\title{
Paying hospitals for quality: can we buy better care?
}

\author{
Jane P Hall, Kees C van Gool
}

$\mathrm{T}$ he idea of paying more for better quality care and paying less (or not at all) for poor quality care appears inherently sensible, and has motivated several attempts to introduce financial incentives for improving the quality of health care. These range from bonus payments to hospitals that meet specified clinical indicators for particular conditions (Queensland and Western Australia are currently implementing such schemes) or paying for structures that encourage quality (including higher payments for achieving accreditation), to penalties for poor quality, such as withholding payment for "never ever events" or shocking medical errors, such as wrong site surgery (as applied in the United Kingdom and by the United States Centers for Medicaid and Medicare Services). ${ }^{1,2}$ Activity-based funding for hospital care is widely used across the world, and quality payments are generally applied as adjustments to the case payments. Financial incentives that reward quality are also applied in primary care, but this article will focus on hospitals.

Two key questions must be considered when thinking about paying for quality. The first is whether the standard case payment is inadequate for providing quality care; that is, whether appropriate quality care, even when supplied efficiently, simply costs more than the case payment. The second is whether the use of rewards (and penalties) will encourage more appropriate or higher quality care. Any use of payments or other incentives obviously requires a clear definition of appropriate quality.

The first question involves the concern that quality could be actively constrained by not paying enough for care. There can be no sensible argument in support of paying too little to achieve appropriate quality; the question is whether it is likely to happen. In Australia, the casemix price paid through activity-based funding is based on the analysis of existing cost data supplied by public hospitals in all states and territories. Unless the present quality of care is significantly lower than appropriate, the national price will therefore reflect adequate care. This could change with technological advances or new evidence about clinical effectiveness. Incremental change is incorporated by regular revisions of casemix classifications and price. Even a major shock in terms of technological progress or additional evidence is likely to be focused on a particular case type, so that the impact on total hospital revenue would be marginal and corrected within a short period of time. While arbitrary cuts can be made by funders to the national price, these would not be a cost-based determination.

The second question is both more interesting and more challenging. Financial incentives that target health care use are generally effective, consistent with economic theory. It should follow that aligning incentives with quality improvement should enhance the quality of care. Evidence collected over the past decade has been disappointing in this respect, ${ }^{3,4}$ and more recent critiques are no more encouraging in terms of their finding improved health outcomes. ${ }^{5}$ One reason for these disappointing findings is that financial incentives are not precise, and there are wide differences in the characteristics and the contexts of the different programs.

\section{Summary}

- Economic theory predicts that changing financial rewards will change behaviour. This is valid in terms of service use; higher costs reduce health care use.

- It should follow that paying more for quality should improve quality; however, the research evidence thus far is equivocal, particularly in terms of better health outcomes. One reason is that "financial incentives" encompass a range of payment types and sizes of reward.

- The design of financial incentives should take into account the desired change and the context of existing payment structures, as well as other strategies for improving quality; further, financial incentives should be fair in rewarding effort.

- Financial incentives may have unintended consequences, including rewarding hospitals for selecting patients with lower risks, diverting attention from the overall patient population to specific conditions, gaming, and "crowding out" or displacing intrinsic motivation.

- Managers and clinicians can only respond to financial incentives if they have the data, tools and skills to effect changes.

- Australia should not adopt widespread use of financial incentives for improving quality in health care without careful consideration of their design and context, the potential for unintended effects (particularly beyond their immediate targets), and evaluation of outcomes. The relative cost-effectiveness of financial incentives compared with, or in concert with, other strategies should also be considered.

For example, incentives may be positive (extra payments) or negative (reducing or withholding payments). The incentives might apply at the hospital level, benefiting the hospital budget as a whole; at a clinical department level, benefiting the departmental budget; or be directed to individual clinicians, in cash or in kind. The size of the incentive ranges from token payments to substantial funding. This would make it difficult to generalise findings about outcomes, even if there were a series of rigorous evaluations. Any design or evaluation should therefore start by considering whether the financial incentive is well targeted and appropriate for the behaviour it is trying to change.

Financial incentives can be applied to hospital-level indicators that encompass all conditions, or be targeted at particular conditions. Targeting a particular condition is more precise, but loses the scope of the entire patient population. Even a high volume condition affects a small proportion of the patient population, so that a targeted program will achieve only a limited overall impact.

Incentives can be directed to reward either better outcomes or improved processes of care. While better health outcomes for patients are undoubtedly the desired end, good quality care does not guarantee a better outcome for the individual. Many factors other than the processes of care influence outcomes, and there is always an element of chance. Further, some outcomes 
may not be experienced until years after care has been delivered.

The economic principle underlying the design of payment schemes is that the funder should reward effort that promotes the interests of the funder. This requires that the hospital, department or clinician is rewarded for what they do and for how they manage factors within their control. Not surprisingly, most financial incentives are therefore applied to improving processes of care. However, to avoid losing sight of the desired better outcomes, evidence that improving the specified processes leads to better outcomes is generally required.

Establishing the evidence base is challenging. Hospitals are complex organisations and hospital care typically involves a variety of clinicians and diagnostic and treatment modalities. The links between incentive, process and health outcome depend on the actions and interactions of many different players.

As stated earlier, a performance payment alone does not achieve anything. Generally, a specific payment is grafted onto an existing payment scheme; and, unsurprisingly, what works in one context or in one organisational and funding model cannot necessarily be extrapolated to another. For example, whether funders and hospitals are operating in a competitive market or in a centrally controlled system will affect how a pay-for-performance model works. Additional payments grafted onto a population-funded model would be expected to have a different impact to paying the same reward as an adjustment of activity-based funding. Further, incentives are complex and encompass more than financial rewards. Professional status, striving for excellence, and effective leadership can each influence behaviour. ${ }^{6}$

Evidence of no benefit is not the same as no evidence of benefit. The first means that there is evidence that an intervention is not effective; the second that the necessary research has not been undertaken. Even without strong evidence of improved outcomes, some people would argue that rewarding good quality processes is, in itself, valuable because quality should be encouraged, and higher quality is more likely to improve than to harm outcomes. However, it is important to first ask whether there could be unintended consequences of payment schemes, and the answer is unequivocally: yes.

The initial effect of a payment incentive is to focus attention. Some reward payments are too small or too cumbersome for potential recipients to claim, making them ineffective. Where the incentive is sufficiently strong, the attention of management will be focused on how to maximise the rewards. While this will improve the measured processes of care, it will also draw attention away from other problems. It is difficult to establish what the opportunity cost will be, but improved performance in one aspect of care may come at the expense of foregone improvements, or even deteriorations, elsewhere.

Incentives can also have perverse effects by encouraging gaming. This can involve changing how treatment processes are counted, such as coding conditions as more complex case types; raising the threshold for recognising hospital-acquired conditions; re-defining a multidisciplinary case conference. This effect can be mitigated by monitoring and auditing processes. One of the more undesirable effects is patient selection: if there is a strong incentive to choose less complex patients, an unintended effect is that hospitals may favour easier cases, so that patients in greater need find it difficult to receive treatment. This is more challenging to monitor.

More significant is the problem of intrinsic and extrinsic motivation, which tends to be little discussed in this context. Intrinsic motivation is complex and powerful, and is vital in health care: care providers are motivated by doing their job well, caring for their patients, and gaining the respect of their peers. Extrinsic motivation relates to behaviour that is driven by external rewards; financial incentives are clearly an important form of extrinsic motivation. Not only do extrinsic motivators lose their effectiveness over time, however, they can "crowd out" or displace intrinsic motivation. As a result, the rewarded behaviour is likely to fade once the reward is removed, even among those who were doing the right thing before the incentive is introduced.

Any discussion of financial incentives should also address the notion of purchasing health gains. This is not as simple as it first seems. Let us assume that the Australian social value, or willingness to pay for a degree of health gain as measured in quality-adjusted life-years (QALYs), is about $\$ 75000$ (a reasonable estimate based on Pharmaceutical Benefits Advisory Committee determinations ${ }^{8}$ ). It does not follow that the Australian health care system should spend $\$ 75000$ for every QALY gained; this would certainly not maximise the health outcomes achieved by its budget. ${ }^{9}$ Specific health gains are delivered at a range of prices; for example, if it cost $\$ 15000$ per QALY for a particular treatment, paying $\$ 75000$ represents an overpayment of $\$ 60000$, or a loss of 4 QALYs. Maximising health outcomes requires starting with the lowest cost per QALY, and adding more expensive treatments until the upper budgetary limit, or the maximum social willingness to pay for a QALY, is reached.

Translated into hospital funding, this means not paying the same price for each unit of health gain. It means paying the efficient price for each treatment for each case type, up to the maximum social willingness to pay. It requires an umpire to set the efficient price for a case type, and a different perspective for determining whether this represents value for money. According to this argument, the price paid should reflect the efficient cost of production, but the volume of cases should be managed separately. The efficient price is intended to provide the incentive for better cost control, particularly in high cost hospitals. It is aimed at improving technical efficiency; that is, maximising the output for a given input.

Finally, getting the incentives to be consistent with the desired ends is only part of the challenge. Managers, clinicians and others involved in health care may be offered the "right" incentives, but, if they lack the skills and tools to identify what and how to effect change, they are powerless to respond. ${ }^{10}$

Financial incentives used alone are a blunt instrument. Their effectiveness will depend on the size and design of the reward or penalty, and also on how they reinforce (or are inconsistent with) other signals in the operating environment. It is therefore not surprising that it is difficult to reach general conclusions about their usefulness. It is even more difficult to reach conclusions about their relative cost-effectiveness compared with alternative approaches. Further, financial incentives have an opportunity cost, diverting funds and attention from other problems. Given what is known and what is still uncertain, it would be unwise to rush into their widespread use as a strategy for improving the quality of health care in Australia. Instead, they should be applied cautiously, with careful consideration of design and context and of the potential for unintended effects, and with evaluation of outcomes.

Competing interests: Jane Hall is a member of the Independent Hospital Pricing Authority (IHPA), but the views expressed here are hers and do not necessarily reflect the views or policy of IHPA.

Provenance: Not commissioned; externally peer reviewed.

(c) 2016 AMPCo Pty Ltd. Produced with Elsevier B.V. All rights reserved. 
1 Eagar K, Sansoni J, Loggie C, et al. A literature review on integrating quality and safety into hospital pricing systems. Wollongong: Australian Health Services Research Institute, University of Wollongong, 2013. http://ro.uow.edu.au/ahsri/385/ (accessed Dec 2015).

2 Scott A, Ouakrim D. Using financial incentives to improve the performance of hospital clinicians: a rapid review. Sydney: Sax Institute, 2011. https://www.saxinstitute.org. au/wp-content/uploads/07_Using-financial-incentives-toimprove-the-performance-of-.pdf (accessed Sept 2015).

3 Rosenthal MB, Frank RG. What is the empirical basis for paying for quality in health care? Med Care Res Rev 2006; 63: 135-157.

4 Christianson J, Leatherman S, Sutherland K. Financial incentives, healthcare providers and quality improvements. A review of the evidence. London: The Health Foundation, 2007; updated 2009. http://www. health.org.uk/publication/financial-incentiveshealthcare-providers-and-quality-improvements (accessed Dec 2015).

5 Cashin C, Chi YL, Smith P, et al. (editors). Paying for performance in health care: implications for health system performance and accountability. Maidenhead (UK): Open University Press, 2014. http://www.euro.who. int/_data/assets/pdf_file/0020/271073/Paying-forPerformance-in-Health-Care.pdf?ua=. (accessed 25 Sept 2015)

6 Kolstad JT. Information and quality when motivation is intrinsic: evidence from surgeon report cards. Am Econ Rev 2013; 103: 2875-2910.
7 Cameron J, Banko KM, Pierce WD. Pervasive negative effects of rewards on intrinsic motivation: the myth continues. Behav Anal 2001; 24: 1-44.

8 George B, Harris A, Mitchell A. Cost-effectiveness analysis and the consistency of decision making: evidence form pharmaceutical reimbursement in Australia (1991-1996). Pharmacoeconomics 2001; 19: 1103-1109.

9 Birch S, Gafni A. Cost effectiveness/utility analyses. Do current decision rules lead us to where we want to be? J Health Econ 1992; 11: 279-296.

10 Damato A. Improving patient outcomes: leveraging data to drive innovation in health care - New South Wales' Activity-Based Funding management portal. BMC Health Serv Res 2015; 15 Suppl 2: Al. 